HBV acquisition, chronic HBV infection and HBV disease. Chronic HBV infection may pose a challenge among HIV infected individuals' eligible for antiretroviral therapy, increasing their risk of rapid HIV-disease progression. Early prevention of HBV infection by vaccinating HIV infected people is recommended. However, in most poor countries HBV vaccination is not routine and those opting for vaccination are rarely tested before vaccination. We sought to determine the prevalence of HBV infection and natural immunity against HBV infection among HIV discordant couples.

Methods The first 949 discordant heterosexual couples screened for eligibility into an HIV pre-exposure prophylaxis study had HBV surface antigen ( $\mathrm{HBs} \mathrm{Ag}$ ) and anti-HBs status determined. CD4 count was also performed for HIV infected potential subjects. None of these participants reported HBV vaccination history. SPSS version 17 software was used for statistical analysis.

Results Of 949 HIV positive subjects, 34.7\% (329) were men thus women were more likely to be the HIV-positive spouse in these HIV-discordant relationships $(\mathrm{p}<0.001) .99$ of 1898 subjects were positive for $\mathrm{HBV}$ sAg, resulting to $5.2 \% \mathrm{HBV}$ prevalence, with men being more likely to be HBV infected compared to women $(p<0.05)$. $40 \%$ (758) of 1898 subjects tested for anti-HBs were immune to $\mathrm{HBV}$, with men being more likely to possess natural protective antibodies against HBV compared to women $(p<0.05)$. Among HIV infected subjects, those with a CD4 count $>250$ cells/ml were more likely to possess immunity against HBV compared to those with a CD4 count $<250$ cells/ml $(p<0.001)$. However, HIV status was not associated with either HBV infection $(p=0.302)$ or immunity against HBV ( $p=0.512)$.

Conclusion Scaling-up routine vaccination after anti-HBs screening in resource limited settings could be cost-effective and easy to roll-out in HIV endemic Sab-Sahara Africa. This is because $40 \%$ of individuals are already immune to HBV. Validation of Anti-HBs rapid screening tests is urgently needed in this population.

\section{P4-S2.02 REGULATORY T CELLS AND FOX P3 LEVELS IN NAIVE AND HAART TREATED HIV-1 INFECTED PATIENTS IN THAILAND}

doi:10.1136/sextrans-2011-050108.514

${ }^{1} \mathrm{~S}$ Sukwit, ${ }^{2} \mathrm{~S}$ Srisurapanon, ${ }^{1} \mathrm{~T}$ Chuenchitra, ${ }^{3} \mathrm{~N}$ Horthongkham, ${ }^{3} \mathrm{R}$ Sutthent. ${ }^{1}$ Armed Forces Research Institute of Medical Sceinces, Bangkok, Thailand; ${ }^{2}$ Srinakharinwirot University, Thailand; ${ }^{3}$ Siriraj Hospital, Mahidol University, Bangkok, Thailand

Background The frequency and expression levels of FoxP3 in regulatory T-cells (T-regs) from advanced-stage HIV-infected patients are controversial. Thus, the aims of this study were to compare these parameters of T-regs from HIV infected patients and from healthy controls.

Methods T-reg population and expression levels of FoxP3 were assessed from 43 HIV-1 infected individuals and 12 healthy controls by using FACs flow cytometry.

Results Minor decrease in frequencies of T-regs was found in both infected groups (naïve and treated) compared with those from healthy controls $(p=0.02, p=0.01$, respectively). In contrast, a significantly increase in the ratios of T-regs: CD4 cells from HIVinfected patients was observed $(p=0.001)$. In addition, FoxP3 expression levels in both treated and untreated HIV-1 infected patients were significantly higher than those in healthy controls $(\mathrm{p}<0.001$ and 0.01 respectively).

Conclusions The decreased number of T-regulatory cells and the increased levels of FoxP3 in T-regs from HIV infected patients were associated with advanced stage of AIDS disease.

\section{P4-S2.03 CALCULATING BMI IN HIV+ FEMALE ADOLESCENTS: A CASE OF SHAPING THE HEALTH OF ADOLESCENTS IN ZIMBABWE: SHAZ! PLUS PROJECT}

doi:10.1136/sextrans-2011-050108.515

D Nhamo. Zimbabwe Aids Prevention Project, University of Zimbabwe, Harare, Zimbabwe

Background Body Mass Index (BMI) is a statistical measure which compares a person's weight and height. It is used to estimate a healthy body weight based on a person's height. This measure is pertinent to use in HIV positives since weight loss is used as a proxy measure for clinical progression of HIV disease in that more weight loss is associated with advancing disease and often precedes clinical symptoms. We sought to measure BMI among HIV+ female adolescents to see if this is the best tool to use in immunecompromised populations.

Methods Data were pulled out from an ongoing RCT entitled SHAZ! Plus. $(\mathrm{N}=650)$. It enrols HIV +ive female adolescents who are out of school. Data were collected at baseline, 6, 12 and 18month follow-up visits, with the project facilitating ongoing care, ART and support to those in need of it. BMI was calculated using Adolphe Quetelet BMI calculator of weight in $\mathrm{kg}$ over height in $\mathrm{m}^{2}$ $\left(\mathrm{kg} / \mathrm{m}^{2}\right)$. BMI prime 25 was also calculated and used to compare the BMI results. Sample of $x x$ used.

Results $64.5 \%$ of the cohort had BMIs falling within normal ranges 18.5 to $25.33 .8 \%$ had BMIs of $<18.5$ and $0.16 \%$ had a BMI of $>25$. $64.5 \%$ had BMI prime that fell within normal ranges of 0.74 and 0.99. 33.8\% had BMI prime of $<0.74$ and $0.16 \%$ had a BMI prime of $>1.00$. The average BMI at baseline was 19.35 Weight ranged from 25 to 70 at baseline, average was 47.71 .

Conclusion Results call for the need to come up with an adjusted BMI index for female adolescents who are immuno-compromised to use as a standard measure for optimal health. BMI however has it's shortcomings since it does not measure the actual body fat. In resource poor settings, a simple tool like weight lost or gained over time might be a good indicator of clinical progression of HIV as opposed to validated tools like the BMI.

\section{Basic sciences poster session 3: ureaplasma, trichomonas and syphilis}

\section{P4-S3.01 MACROLIDE-RESISTANCE TESTING AND MOLECULAR SUBTYPING OF TREPONEMA PALLIDUM STRAINS FROM SOUTH AFRICA}

doi:10.1136/sextrans-2011-050108.516

${ }^{1} E$ Muller, ${ }^{2} \mathrm{G}$ Paz-Bailey, ${ }^{1} \mathrm{D}$ Lewis. ${ }^{1}$ National Institute for Communicable Diseases, National Health Laboratory Service, Johannesburg, South Africa; ${ }^{2}$ Del Valle University of Guatemala, Guatemala

Objectives To determine whether the main $23 \mathrm{~S}$ rRNA mutation (A2058G) that confers macrolide resistance in Treponema pallidum is present among DNA obtained from syphilitic ulcers in South Africa and to determine the strain subtype distribution using molecular methods.

Methods DNA was re-extracted from sixty ulcer swabs, previously shown to contain $T$ pallidum DNA by in-house real-time multiplex PCR assay, using a MagNA Pure Compact DNA extraction system (Roche, Germany). The genital ulcer specimens were collected in South Africa between 2005 and 2009 during either national microbiological surveillance activities or as part of large episodic acyclovir treatment trial (HSV4294). The re-extracted DNA was screened for the A2058G point mutation in the peptidyltransferase region of the 23S rRNA subunit using a rapid PCR-based restriction digest assay. 